\title{
Locally Advanced Carcinoma of the Uterine Cervix: Current Issues in Developing Countries and Future Research
}

\section{Saikat Das}

Department of Radiation Oncology, Christian Medical College and Hospital Vellore, India

*Corresponding author: Saikat Das, Department of Radiation Oncology, Christian Medical College and Hospital Vellore, India, Tel: 914162282046, 919597235339; Email: saikat@cmcvellore.ac.in

Received date: Aug 17, 2015, Accepted date: Sep 14, 2015, Publication date: Sep 18, 2015

Copyright: (c) 2015 Das S. This is an open-access article distributed under the terms of the Creative Commons Attribution License; which permits unrestricted use; distribution; and reproduction in any medium; provided the original author and source are credited.

\begin{abstract}
Locally advanced carcinoma of the uterine cervix is a major public health problem in developing countries. In spite of advancement in chemoradiation therapy, the outcome of locally advanced disease remains suboptimal. Newer diagnostic methods, treatment approaches and technology to evaluate the response needs to be evaluated in this condition. Some of the current issues of management of cervix cancer in developing countries are discussed in this present review. The direction of future research in imaging and neoadjuvant therapy are highlighted.
\end{abstract}

Keywords: Carcinoma of uterine cervix; Chemoradiation; Neoadjuvant therapy

\section{Introduction}

Carcinoma of the uterine cervix is one of the most common cancers in developing countries like India. More than $80 \%$ patients present in a fairly advanced stage [1]. According to the GLOBOCAN statistics, the estimated incidence of cervical cancer in the world is 528000 per year with 266000 annual deaths attributed to cervical cancer (approximately $50 \%$ ) [2]. India alone contributes to $23 \%$ of the total global cases annually. With an annual 67000 deaths, cervical cancer remains one of the major causes of cancer related mortality in India [3]. The staging system proposed by International Federation of Obstetrics and Gynecology (FIGO) is widely followed and correlate well with prognosis [4]. Briefly stage I consists of disease confined to the cervix (IA: microscopic and IB: clinically demonstrable lesion). Stage II disease extends beyond the cervix but not involving pelvic side wall or lower third of vagina.

Involvement of these later structures including hydronephrosis or non-functioning kidney is classified as stage III disease. Stage IV disease extends well beyond the pelvis or involves the mucosa of the bladder or rectum [4]. FIGO system can be followed even in resource constrained situation and is a robust system for predicting survival even though it does not take into account other prognostic factors like endometrial extension, stromal invasion, lympho-vascular extension or radiological nodal involvement [5]. Radiotherapy is the mainstay of treatment for locally advanced cancer of the cervix (stage IIB to IVA). Radiotherapy consists of combination of external beam radiotherapy and brachytherapy to deliver adequate tumoricidal dose to the gross and microscopic tumor [6]. In conventional radiotherapy, target volume encompasses the true pelvis including the primary tumor, pelvic and lower para-aortic nodes. External beam radiotherapy is followed by brachytherapy to boost the primary tumor. In brachytherapy, a very high dose can be delivered to a small volume sparing the normal organs.

\section{Radiotherapy in carcinoma of the cervix}

Various retrospective series have shown that $40-50 \%$ disease control rate can be achieved at 5 years for locally advanced carcinoma cervix with radiotherapy alone. [1]. Montana et al., reported disease free survival at 5 year for IB, IIA, IIB, IIIA and IIIB as $83 \%, 76 \%, 62 \%, 26 \%$ and $35 \%$ respectively [7] . Retrospective outcome analysis from India, reported disease free survival of $56 \%$ and $40 \%$ at 8 years for stage IIB and IIIB respectively with radical radiotherapy. [1]. In a more recent study Azad et al., reported a 3 year disease free survival of $80 \%, 70 \%$, $40 \%$ and $11 \%$ for stage I, II, III and IV cervical cancer respectively by radiotherapy alone [6]. Therefore, for locally advanced cervical cancer, treatment outcome with radiation therapy is suboptimal. Till the beginning of this century radiotherapy alone was the standard of care. Pattern of care study in United States showed that almost $70 \%$ of locally advanced cervical cancer patients were treated with radical radiotherapy between 1996-1999 [8].

\section{Concurrent chemoradiation in carcinoma of the cervix}

In 1999, results of several large randomized trials of concurrent chemotherapy with radiation changed the standard of care for advanced carcinoma cervix. Whereas, the studies involving neoadjuvant chemotherapy followed by radiotherapy, failed to show desired benefit, there was significant reduction in risk of recurrence with concurrent chemoradiotherapy. Pattern of care study, showed the change of practice of management of locally advanced cervical cancer after 1999, with as many as $63 \%$ patients receiving chemoradiation [8]. Addition of chemotherapy with radiation increased the locoregional disease control and survival. Various regimens of chemotherapy were tried during this period which can be classified into platinum based (cisplatin alone or in combination) and non-platinum based. Several trials have successfully investigated the role of addition of chemotherapy to radiotherapy [9-13].This was followed by National Cancer Institute alert urging clinicians to use chemoradiation for cervical cancer [14]. Majority of the studies showed survival benefit, which was further confirmed by Cochrane meta-analysis [15]. The meta-analysis results showed that the addition of chemotherapy to radiotherapy leads to a $12 \%$ increase in overall survival [15]. An 
updated meta-analysis reducing the heterogeneity of the earlier analysis compared data from 13 trials which compared chemo radiotherapy with same radiotherapy. The 5 year survival benefit with chemoradiation was $6 \%$ (hazard ratio $0.81, \mathrm{p}<0.001$ ) [16]. There was a suggestion that magnitude of the survival benefit was more for earlier stage disease [16]. Even though chemoradiation became the standard of care, the evidence of benefit of chemotherapy for advanced stage disease was not so strong and overall survival for such advanced, bulky disease still remains suboptimal.

\section{Chemoradiation in locally advanced carcinoma cervix in developing countries}

Chemoradiation markedly increased the hematological and gastrointestinal toxicity and overall complexity of the treatment [8]. Higher incidence of acute toxicities, poor nutritional status of patients makes it difficult to implement it in developing countries. Moreover, it remained uncertain whether patients with locally advanced disease actually benefit from chemo-radiotherapy [17]. Few Indian studies have evaluated the role of chemoradiation in locally advanced cervical cancer. Negi et al. conducted a prospective randomized study of chemoradiation vs. radiation alone of 102 patients with stages IIA-IVA cervical cancer. This study did not show any benefit of concurrent chemoradiation compared to radiation alone [18]. In another randomized trial, reported by Mitra et al. no statistically significant increase in overall or disease free survival was reported with chemo radiotherapy [19]. Another large randomized trial from India, reported by Srivastava et al. also did not show a survival benefit with concurrent chemoradiotherapy compared to radiation alone ( $58 \%$ vs $60 \%$ ) in a group of 305 patients with stage Ib-IVa cervical cancer [20]. In fact, the role of chemoradiotherapy in locally advanced cervical cancer especially in the context of developing countries has been questioned keeping the risk of combination regimen and overall benefit [17]. This paradoxical effect observed in advanced tumors in developing countries has been attributed to the increased acute toxicity resulting in more frequent treatment breaks with resultant increase in overall treatment time, accelerated repopulation [17]. In developing counties like India, particularly due to prevailing socio-economic conditions, it is very difficult to administer the optimum five cycles of concurrent chemotherapy as recommended by earlier GOG protocol [21]. Therefore, newer treatment modalities, in addition to the present standard of care of concurrent chemoradiotherapy, need to be explored for better tumor control in locally advanced disease.

\section{Role of neoadjuvant chemotherapy}

One of the approaches adopted was to add neoadjuvant chemotherapy prior to the loco-regional treatment. Neoadjuvant chemotherapy effectively reduces the tumor size, eliminates micrometastasis and down stage the tumor [22]. However, the significance of these benefits in terms of survival has not been proven conclusively in the literature, especially in locally advanced disease [23]. Interestingly, from the meta-analysis of neoadjuvant chemotherapy in cervical cancer, it was evident that, trials that gave more intensive chemotherapy in terms of a shorter cycle or higher dose intensity, resulted in better overall survival. Therefore, modification of existing neoadjuvant protocols is required to improve the therapeutic outcome. Dose dense chemotherapy is one of the modifications suggested in the literature [24,25]. One of the other approaches is chemopotentiation of chemotherapy with the addition of low dose radiation. Recent radiobiological investigations have shown that ultra- fractionated low dose of radiotherapy (0.5-1 Gy) can increase the efficacy of cell cycle specific chemotherapy [26-29]. At this dose, radiation leads to arrest of the proliferating cells in G2 phase by inducing DNA repair mechanism. Addition of G2 phase specific chemotherapy can lead to increased cell kill [30]. This phenomenon is called chemopotentiation and it has been shown to be a clinically feasible and effective approach in head and neck cancer, glioblastoma, breast cancer, non-small cell lung cancer, endometrial cancer and pancreatic cancer [31-37]. Combination of low dose radiation with chemotherapy to increase the efficacy is based on radiobiological synergism due to chemopotentiation. Modification of neoadjuvant chemotherapy by dose dense approach or by chemopotentation by low dose radiation has been reported in the literature to improve the therapeutic outcome $[24,25,30]$. Das et al. reported $84 \%$ disease control at 3 years in cervix cancer with ultra-fractionated low dose radiation and chemotherapy followed by chemoradiation [38]. The role of neoadjuvant chemotherapy especially in the context of chemoradiation requires further evaluation.

\section{Advancement in imaging technology}

Use of magnetic resonance imaging (MRI) especially functional MRI like diffusion weighted imaging (DWI) has improved the diagnostic and therapeutic efficacy in cervix cancer. MRI enabled image guided adaptive brachytherapy has improved the outcome as shown in various studies [39,40]. Escalation of dose with sparing of the normal structures is possible by this approach which can deliver significantly high dose to the tumor. Diffusion weighted MRI has improved the diagnostic accuracy of malignancies in gynecological cancers. MRI can help to stage the disease more accurately due to better soft tissue delineation especially in the context of parametrial and lower uterine segment involvement. It can also help to differentiate radiation induced tissue changes from residual disease [41-45]. Recently it has been shown that Positron Emission Tomography (PET) scan can improve the diagnostic accuracy especially in case of recurrent and metastatic disease [46], however, the role of PET scan in cervix cancer is still translational.

\section{Conclusion}

In conclusion, advanced carcinoma of the uterine cervix continues to be a therapeutic challenge in developing countries. Use of modern radiation therapy technology especially adaptive radiation with image guidance, newer chemotherapy approaches and functional imaging can improve the therapeutic outcome of this condition. In future, the correlation of radiological or imaging markers with pathological prognostic markers should be investigated.

\section{References}

1. Shrivastava S, Mahantshetty U, Engineer R, Tongaonkar H, Kulkarni J, et al. (2013) Treatment and outcome in cancer cervix patients treated between 1979 and 1994: a single institutional experience. J Cancer Res Ther 9: 672-679.

2. Ferlay JSI, Ervik M, Dikshit R, Eser S, Mathers C, et al. (2013) Cancer Incidence and Mortality Worldwide: IARC CancerBase No. 11 [Internet]. Lyon, France: International Agency for Research on Cancer: 2012.

3. Nandakumar A, Ramnath T, Chaturvedi M (2009) The magnitude of cancer cervix in India. Indian J Med Res 130: 219-221.

4. Zalewski K, Doniec J, Baranowski W, Bidzinski M (2010) [Revised FIGO staging systems for gynecologic malignancies--2009 update]. Ginekol Pol 81: 778-782. 
5. Prasad TV, Thulkar S, Hari S, Sharma DN, Kumar S (2014) Role of computed tomography (CT) scan in staging of cervical carcinoma. Indian J Med Res 139: 714-719.

6. Azad SK, Choudhary V (2010) Treatment results of radical radiotherapy of carcinoma uterine cervix using external beam radiotherapy and high dose rate intracavitary radiotherapy. J Cancer Res Ther 6: 482-486.

7. Montana GS, Hanlon AL, Brickner TJ, Owen JB, Hanks GE, et al. (1995) Carcinoma of the cervix: patterns of care studies: review of 1978, 1983, and 1988-1989 surveys. Int J Radiat Oncol Biol Phys 32: 1481-1486.

8. Eifel PJ (2006) Chemoradiotherapy in the treatment of cervical cancer. Semin Radiat Oncol 16: 177-185.

9. Keys HM, Bundy BN, Stehman FB, Okagaki T, Gallup DG, et al. (2003) Radiation therapy with and without extrafascial hysterectomy for bulky stage IB cervical carcinoma: a randomized trial of the Gynecologic Oncology Group. Gynecol Oncol 89: 343-353.

10. Eifel PJ, Winter K, Morris M, Levenback C, Grigsby PW, et al. (2004) Pelvic Irradiation With Concurrent Chemotherapy Versus Pelvic and Para-Aortic Irradiation for High-Risk Cervical Cancer: An Update of Radiation Therapy Oncology Group Trial (RTOG) 90-01. J Clin Oncol 22: 872-880.

11. Whitney CW, Sause W, Bundy BN, Malfetano JH, Hannigan EV, et al. (1999) Randomized Comparison of Fluorouracil Plus Cisplatin Versus Hydroxyurea as an Adjunct to Radiation Therapy in Stage IIB-IVA Carcinoma of the Cervix With Negative Para-Aortic Lymph Nodes: A Gynecologic Oncology Group and Southwest Oncology Group Study. Journal of Clinical Oncology 17: 1339.

12. Rose PG, Ali S, Watkins E, Thigpen JT, Deppe G, et al. (2007) Long-Term Follow-Up of a Randomized Trial Comparing Concurrent Single Agent Cisplatin, Cisplatin-Based Combination Chemotherapy, or Hydroxyurea During Pelvic Irradiation for Locally Advanced Cervical Cancer: A Gynecologic Oncology Group Study. Journal of Clinical Oncology 25: 2804-2810.

13. Cetina L, Rivera L, Hinojosa J, Poitevin A, Uribe J, et al. (2006) Routine management of locally advanced cervical cancer with concurrent radiation and cisplatin. Five-year results. BMC Womens Health 6: 3.

14. Kantardzic N (2010) Concurrent chemoradiation for cervical cancer: results of five randomized trials. Med Arh 64: 368-370.

15. Green J, Kirwan J, Tierney J, Vale C, Symonds P, et al. (2001) Concomitant chemotherapy and radiation therapy for cancer of the uterine cervix. Cochrane Database Syst Rev: CD002225.

16. Chemoradiotherapy for Cervical Cancer Meta-analysis Collaboration (CCCMAC) (2010) Reducing uncertainties about the effects of chemoradiotherapy for cervical cancer: individual patient data metaanalysis. Cochrane Database Syst Rev : CD008285.

17. Datta NR, Agrawal S (2006) Does the Evidence Support the Use of Concurrent Chemoradiotherapy as a Standard in the Management of Locally Advanced Cancer of the Cervix, Especially in Developing countries? Clinical Oncology18: 306-312.

18. Negi RR, Gupta M, Kumar M, Gupta MK, Seam R, et al. (2010) Concurrent chemoradiation in locally advanced carcinoma cervix patients. J Cancer Res Ther 6: 159-166.

19. Mitra D, Ghosh B, Kar A, Basu S, Deb AR, et al. (2006) Role of chemoradiotherapy in advanced carcinoma cervix. J Indian Med Assoc 104: 432, 434, 436 passim.

20. Srivastava K, Paul S, Chufal KS, Shamsundar SD, Lal P, et al. (2013) Concurrent chemoradiation versus radiotherapy alone in cervical carcinoma: A randomized phase III trial. Asia Pac J Clin Oncol 9: 349-356.

21. Harjani RR, Janaki MG, Somashekhar M, Ponni A, Alva RC, et al. (2014) Feasibility of Concurrent Chemoradiation in Cervical Cancer Patients From Rural Background. Clinical Ovarian and Other Gynecologic Cancer 7: 29-32.

22. Osman M (2014) The role of neoadjuvant chemotherapy in the management of locally advanced cervix cancer: a systematic review. Oncol Rev 8: 250.
23. Neoadjuvant Chemotherapy for Locally Advanced Cervical Cancer Metaanalysis Collaboration (2003) Neoadjuvant chemotherapy for locally advanced cervical cancer: a systematic review and meta-analysis of individual patient data from 21 randomised trials. Eur J Cancer 39: 2470-2486.

24. McCormack M, Kadalayil L, Hackshaw A, Hall-Craggs MA, Symonds RP, et al. (2013) A phase II study of weekly neoadjuvant chemotherapy followed by radical chemoradiation for locally advanced cervical cancer. Br J Cancer 108: 2464-2469.

25. Singh RB, Chander S, Mohanti BK, Pathy S, Kumar S, et al. (2013) Neoadjuvant chemotherapy with weekly paclitaxel and carboplatin followed by chemoradiation in locally advanced cervical carcinoma: a pilot study. Gynecol Oncol 129: 124-128.

26. Joiner MC, Lambin P, Malaise EP, Robson T, Arrand JE, et al. (1996) Hypersensitivity to very-low single radiation doses: its relationship to the adaptive response and induced radioresistance. Mutat Res 358: 171-183.

27. Joiner MC, Lambin P, Marples B (1999) Adaptive response and induced resistance. C R Acad Sci III 322: 167-175.

28. Joiner MC, Marples B (1992) Does induced repair determine the lethal effects of very low radiation doses? BJR Suppl 24: 74-78.

29. Joiner MC, Marples B, Lambin P, Short SC, Turesson I (2001) Low-dose hypersensitivity: current status and possible mechanisms. Int J Radiat Oncol Biol Phys 49: 379-389.

30. Prasanna A, Ahmed MM, Mohiuddin M, Coleman CN (2014) Exploiting sensitization windows of opportunity in hyper and hypo-fractionated radiation therapy. J Thorac Dis 6: 287-302.

31. Arnold SM, Regine WF, Ahmed MM, Valentino J, Spring P, et al. (2004) Low-dose fractionated radiation as a chemopotentiator of neoadjuvant paclitaxel and carboplatin for locally advanced squamous cell carcinoma of the head and neck: results of a new treatment paradigm. Int J Radiat Oncol Biol Phys 58: 1411-1417.

32. Regine WF, Hanna N, Garofalo MC, Doyle A, Arnold S, et al. (2007) Low-dose radiotherapy as a chemopotentiator of gemcitabine in tumors of the pancreas or small bowel: a phase I study exploring a new treatment paradigm. Int J Radiat Oncol Biol Phys 68: 172-177.

33. Nardone L, Valentini V, Marino L, De Santis MC, Terribile D, et al. (2012) A feasibility study of neo-adjuvant low-dose fractionated radiotherapy with two different concurrent anthracycline-docetaxel schedules in stage IIA/B-IIIA breast cancer. Tumori 98: 79-85.

34. Valentini V, Massaccesi M, Balducci M, Mantini G, Micciché F, et al. (2010) Low-dose hyperradiosensitivity: is there a place for future investigation in clinical settings? Int J Radiat Oncol Biol Phys 76: 535-539.

35. Balducci M, Chiesa S, Diletto B, D'Agostino GR, Mangiola A, et al. (2012) Low-dose fractionated radiotherapy and concomitant chemotherapy in glioblastoma multiforme with poor prognosis: a feasibility study. Neuro Oncol 14: 79-86.

36. Mantini G, Valentini V, Meduri B, Margaritora S, Balducci M, et al. (2012) Low-dose radiotherapy as a chemo-potentiator of a chemotherapy regimen with pemetrexed for recurrent non-small-cell lung cancer: a prospective phase II study. Radiother Oncol 105: 161-166.

37. Wrenn DC, Saigal K, Lucci JA 3rd, Pearson MJ, Simpkins F, et al. (2011) A Phase I Study using low-dose fractionated whole abdominal radiotherapy as a chemopotentiator to full-dose cisplatin for optimally debulked stage III/IV carcinoma of the endometrium. Gynecol Oncol 122: 59-62.

38. Das S, Subhashini J, Rami Reddy JK, KantiPal S, Isiah R, et al. (2015) Low-dose fractionated radiation and chemotherapy prior to definitive chemoradiation in locally advanced carcinoma of the uterine cervix: Results of a prospective phase II clinical trial. Gynecol Oncol 138: 292-298.

39. Lindegaard JC, Fokdal LU, Nielsen SK, Juul-Christensen J, Tanderup K (2013) MRI-guided adaptive radiotherapy in locally advanced cervical cancer from a Nordic perspective. Acta Oncol 52: 1510-1519.

40. Pötter R, Georg P, Dimopoulos JC, Grimm M, Berger D, et al. (2011) Clinical outcome of protocol based image (MRI) guided adaptive brachytherapy combined with $3 \mathrm{D}$ conformal radiotherapy with or 
Citation: Das S (2015) Locally Advanced Carcinoma of the Uterine Cervix: Current Issues in Developing Countries and Future Research. J Nucl Med Radiat Ther 6: 247. doi:10.4172/2155-9619.1000247

Page 4 of 4

without chemotherapy in patients with locally advanced cervical cancer Radiother Oncol 100: 116-123.

41. Alvarez Moreno E, Jimenez de la Peña M, Cano Alonso R (2012) Role of New Functional MRI Techniques in the Diagnosis, Staging, and Followup of Gynecological Cancer: Comparison with PET-CT. Radiol Res Pract 2012: 219546

42. Birlik B, Obuz F, Elibol FD, Celik AO, Sokmen S, et al. (2015) Diffusionweighted MRI and MR- volumetry - in the evaluation of tumor response after preoperative chemoradiotherapy in patients with locally advanced rectal cancer. Magn Reson Imaging 33: 201-212.

43. Fu ZZ, Peng Y, Cao LY, Chen YS, Li K, et al. (2015) Value of apparent diffusion coefficient (ADC) in assessing radiotherapy and chemotherapy success in cervical cancer. Magn Reson Imaging 33: 516-524.
44. Hameeduddin A, Sahdev A (2015) Diffusion-weighted imaging and dynamic contrast-enhanced MRI in assessing response and recurrent disease in gynaecological malignancies. Cancer Imaging 15: 3.

45. Ho KC, Lin G, Wang JJ, Lai CH, Chang CJ, et al. (2009) Correlation of apparent diffusion coefficients measured by $3 \mathrm{~T}$ diffusion-weighted MRI and SUV from FDG PET/CT in primary cervical cancer. Eur J Nucl Med Mol Imaging 36: 200-208.

46. Lee SI, Catalano O, Dehdashti F (2015) Evaluation of gynecologic cancer with MR imaging, 18F-FDG PET/CT, and PET/MR imaging. J Nucl Med 56: $436-443$. 